\title{
New applications of non-hermitian random matrices*
}

\author{
A.Zabrodin ${ }^{\dagger}$ \\ October 2002
}

\begin{abstract}
We discuss recently discovered links of the statistical models of normal random matrices to some important physical problems of pattern formation and to the quantum Hall effect. Specifically, the large $N$ limit of the normal matrix model with a general statistical weight describes dynamics of the interface between two incompressible fluids with different viscousities in a thin plane cell (the SaffmanTaylor problem). The latter appears to be mathematically equivalent to the growth of semiclassical 2D electronic droplets in a strong uniform magnetic field with localized magnetic impurities (fluxes), as the number of electrons increases. The equivalence is most easily seen by relating the both problems to the matrix model.
\end{abstract}

\section{ITEP-TH-46/02}

*Talk given at TH-2002, Paris, UNESCO, July 22-27 2002

†Institute of Biochemical Physics, Kosygina str. 4, 119991 Moscow, Russia and ITEP, Bol. Cheremushkinskaya str. 25, 117259 Moscow, Russia 


\section{Introduction}

The subject of the theory of random matrices is a random matrix $\mathrm{M}$ distributed with some probability measure $d \mu(\mathrm{M})$. Typically, one is interested in the distribution of eigenvalues and correlations between them as size of the matrix, $N$, tends to infinity.

The range of physical applications of this theory is enormously vast, with the role played by the matrix $\mathrm{M}$ being very different. In complex systems or systems with disorder $M$ turns out to be a good substitute for the Hamiltonian or transfer matrix. Physical characteristics of the system are obtained via averaging over one or another ensemble of large matrices. Most extensively employed and well-studied are ensembles of hermitian matrices. In more recent applications to statistical models on random lattices and to string theory the key element is a set of graphs in the diagrammatic expansion of random matrix integrals while $M$ has no physical meaning by itself. For different aspects of random matrix theory and related topics see e.g. [1, 2, 3].

Complex non-hermitian random matrices are employed in physics too. (A list of the relevant physical problems and corresponding references can be found in, e.g., [4].) New applications we are going to discuss are related to the distribution of their eigenvalues. To be specific, we consider the model of normal random matrices, i.e., such that $\mathrm{M}$ commutes with its hermitian conjugate, though similar results may hold for other ensembles. Eigenvalues of normal matrices are in general complex numbers. When $N$ becomes large, they densely fill a domain in the complex plane, the support of eigenvalues, with the mean density outside it being zero. The shape of this domain is determined by the probability measure and by the size of the matrix. As $N$ increases, the domain grows (see Fig. 1). The growth law is our main concern in this paper.

For simplicity we assume that the support of eigenvalues, $D$, is a connected domain. Let the size of the matrix grow linearly in time $t: N \sim t$. Then the support of eigenvalues grows in such a way that the normal velocity of the boundary is

$$
\vec{v}(z)=\operatorname{grad} \varphi(z), \quad z \in \partial D
$$

where $\varphi$ is a function such that

$$
\begin{cases}\Delta \varphi(z)=0 & z \in \mathbf{C} \backslash D \\ \varphi(z) \sim \log |z| & z \rightarrow \infty \\ \varphi(z)=0 & z \in \partial D\end{cases}
$$

Here $\Delta=\partial_{x}^{2}+\partial_{y}^{2}=4 \partial_{z} \partial_{\bar{z}}$ is the Laplace operator. We employ the complex notation $z=x+i y$. So, the dynamics of the boundary is governed by the function $\varphi$ which is harmonic in the exterior of $D$ with a source at infinity, and vanishes on the boundary. The solution of the boundary problem (2) is unique: $\varphi(z)=\log |w(z)|$, where $w(z)$ is the conformal map from the exterior of the domain $D$ onto the exterior of the unit circle

such that $\infty$ is mapped to $\infty$. Such a map exists by virtue of the Riemann mapping theorem.

This dynamics is realized in various physical problems, classical and quantum. First, it is pertinent to viscous flows and other pattern formation processes when the normal 


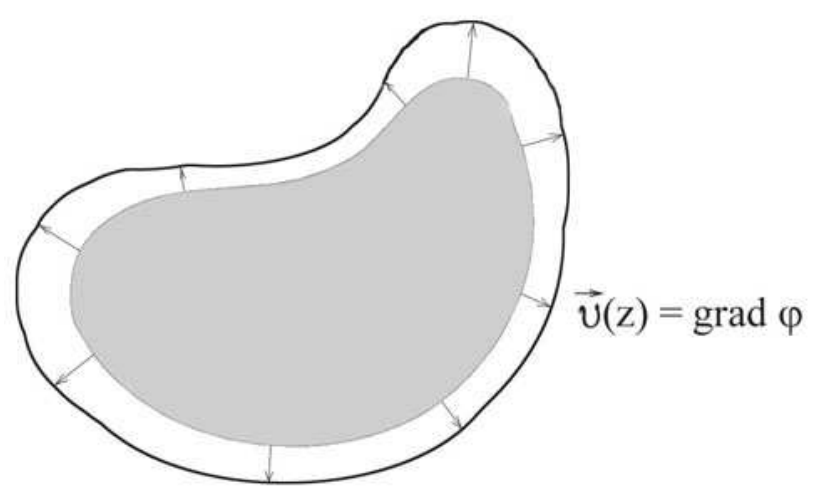

Figure 1: Growth of the support of eigenvalues.

velocity of the moving front is proportional to the gradient of a harmonic field (see, e.g., [5]). This mechanism is known as Darcy's law. The droplet of eigenvalues behaves like an incompressible fluid with negligible viscosity (say water) surrounded by a viscous fluid (say oil), two fluids being confined in a thin plane gap (the Hele-Shaw cell). Oil is withdrawn at infinity at a constant rate while water is injected. In this context, the function $\varphi$ is identified with the pressure $P$ in the viscous fluid with the opposite sign: $\varphi=-P$. In water, the pressure can be set to 0 . The condition that $P=0$ on the interface amounts to neglecting the surface tension effects. This idealization is good until the curvature of the interface becomes large. When the surface tension is small enough, the dynamics becomes unstable. The moving interface develops many fingers, they split into new ones, and in a sufficiently long time the water droplet looks like a fractal. In the literature, this phenomenon is refered to as the Saffman-Taylor fingering. This growth process was linked to the matrix model in [6].

As was recently pointed out in [0], the same growth law applies to semiclassical dynamics of an electronic droplet confined in a plane on the lowest Landay level of a strong magnetic field. This suggests applications to the Quantum Hall effect. It turns out that the shape of the electronic droplet is sensitive to magnetic fluxes localized well away from it. As one changes degeneracy of the level to increase the number of electrons in the droplet, its shape evolves in accordance with the Darcy law, thus showing up fingering instabilities. This phenomenon is purely quantum. Like the Aharonov-Bohm effect, it is caused by quantum interference. The function $\varphi$ in this case has no obvious physical interpretation. The characteristic scale of this phenomenon is less than that of the Saffman-Taylor fingering by a factor of $10^{9}$. Remarkably, the matrix model provides a unified mathematical treatment of the both phenomena.

From mathematical side, it is also worth noting that calculation of certain expectation values and correlation functions of normal random matrices provides a constructive proof of some important mathematical statements in the inverse potential problem and the Dirichlet boundary problem proved in a different way in 87 .

At last, the normal matrix model is known to be integrable. Its partition function is a tau-function of an integrable hierarchy of partial differential equations. Although we do not discuss integrability matters in this paper, let us point out that the above physical problems thus possess a hidden integrable structure. 


\section{Partition function of normal random matrices}

A matrix $\mathrm{M}$ is called normal if $\left[\mathrm{M}, \mathrm{M}^{\dagger}\right]=0$, so that $\mathrm{M}$ and $\mathrm{M}^{\dagger}$ can be simultaneously diagonalized. The model of normal random matrices was introduced in [9]. The integrable structure of the model is quite similar to that of the more widely known model of two hermitian random matrices [10] but physical interpretation is very different.

The partition function is

$$
Z_{N}=\int_{\text {normal }} d \mu_{0}(\mathrm{M}) e^{\frac{1}{\hbar} \operatorname{tr} V\left(\mathrm{M}, \mathrm{M}^{\dagger}\right)}
$$

where $\hbar$ is a parameter and $V$ is a real-valued function. The measure $d \mu_{0}$ is induced from the standard flat metric on the space of all complex matrices. To introduce coordinates in the subspace of normal matrices, one makes use of the decomposition $\mathrm{M}=\mathrm{UZZ}^{\dagger}$ of a normal matrix $\mathrm{M}$, where $\mathrm{U}$ is a unitary matrix and $\mathrm{Z}=\operatorname{diag}\left(z_{1}, \ldots, z_{N}\right)$ is the diagonal matrix of eigenvalues of the $M$. The measure is then given by

$$
d \mu_{0}(\mathrm{M})=\frac{d \mu_{\mathcal{U}(N)}(\mathrm{U})}{N ! \operatorname{Vol}(\mathcal{U}(N))}\left|\Delta_{N}(z)\right|^{2} \prod_{i=1}^{N} d^{2} z_{i}
$$

where $d \mu_{\mathcal{U}(N)}$ is the Haar measure on the unitary group $\mathcal{U}(N)$, and $\Delta_{N}(z)=\prod_{i>j}^{N}\left(z_{i}-z_{j}\right)$ is the Vandermonde determinant. The partition function is, therefore, written as the following integral over eigenvalues:

$$
Z_{N}=\frac{1}{N !} \int\left|\Delta_{N}(z)\right|^{2} \prod_{j=1}^{N}\left(e^{\frac{1}{\hbar} V\left(z_{j}\right)} d^{2} z_{j}\right)
$$

(For notational simplicity we shall write $V(z)$ instead of $V(z, \bar{z})$.) This quantity has two important interpretations.

One of them is the Coulomb gas picture [1]. Writing $Z_{N}=\frac{1}{N !} \int e^{\mathcal{E}\left(z_{i}\right)} \prod_{j=1}^{N} d^{2} z_{j}$ where

$$
\mathcal{E}=\underbrace{\sum_{i \neq j} \log \left|z_{i}-z_{j}\right|}_{2 \mathrm{D} \text { Coulomb energy }}+\hbar^{-1} \underbrace{\sum_{i} V\left(z_{i}\right)}_{\text {potential }}
$$

we see that $Z_{N}$ is the partition function of the $2 \mathrm{D}$ Coulomb gas in the external potential. Another one is the Quantum Hall picture suggested in [7].

Quantum Hall picture. Consider spin- $\frac{1}{2}$ electrons on the plane in a non-uniform magnetic field $B$. The Pauli hamiltonian is

$$
H=\frac{1}{2 m}\left((-i \hbar \nabla-\vec{A})^{2}-\hbar \sigma_{3} B\right)
$$

where $\vec{A}$ is the vector potential. If the magnetic field is uniform, the spectrum consists of equidistant Landau levels, each level being highly degenerate. The lowest level is very special. Due to a hidden supersymmetry of the problem, it can be found exactly and 
remains highly degenerate even for arbitrary non-uniform field $B$ [12]. The energy of this level equals 0 while the degeneracy equals the integer part of the total magnetic flux $\Phi=\int B(z) d^{2} z$ in units of the flux quantum $\Phi_{0}=2 \pi \hbar$ (we set $e=c=1$ ). One-particle states on the lowest level can be found explicitly. In the gauge $A_{x}=\frac{1}{2} \partial_{y} V, A_{y}=-\frac{1}{2} \partial_{x} V$ they are

$$
\psi_{n}(z)=P_{n}(z) \exp \left(\frac{V(z)}{2 \hbar}\right)
$$

where $B(z)=-\frac{1}{2} \Delta V(z)$. Here $P_{n}=z^{n}+$ [terms of lower degree] are holomorphic polynomials of any degree which is less than the degeneracy of the level [12].

Neglecting interactions between electrons, the wave function of $N$ particles on the lowest level is the Jastrow determinant: $\Psi_{N} \sim \frac{1}{\sqrt{N !}} \operatorname{det} \psi_{n}\left(z_{m}\right)$, and so

$$
\left|\Psi_{N}\right|^{2}=\frac{1}{N !}\left|\Delta_{N}\left(z_{i}\right)\right|^{2} e^{\frac{1}{\hbar} \sum_{n} V\left(z_{n}\right)}
$$

coincides with the statistical weight of normal random matrices expressed through eigenvalues. The partition function (स) is, in this context, the normalization factor of the $N$-particle wave function: $\int\left|\Psi_{N}\right|^{2} \prod_{i} d^{2} z_{i}=Z_{N}$. The mean density of electrons coincides with the expectation value of the density of eigenvalues in the matrix model:

$$
N Z_{N}^{-1} \int\left|\Psi_{N}\left(z, \xi_{1}, \ldots, \xi_{N-1}\right)\right|^{2} \prod_{i=1}^{N-1} d^{2} \xi_{i}=\left\langle\operatorname{tr} \delta^{(2)}(z-\mathrm{M})\right\rangle
$$

Similarly, multiparticle correlation functions are identified with multipoint correlation functions of densities.

All the above relations are exact at any finite $N$. As $\hbar$ becomes small and $N$ large, one approaches a semiclassical regime. However, the semiclassical properties of the system are quite unusual. On the one hand, the density distribution acquires a well-defined edge, and one can speak about a well localized electronic droplet which behaves like an incompressible fluid. On the other hand, in this specific semiclassical regime, quantum effects are by no means negligible. Quite the reverse, they become rather strong if not dominant. In fact there is no surprize here because the semiclassical limit we are speaking about is not the usual one which would require excitations of higher energy levels. In our "semiclassical" limit all particles occupy the lowest level, so the droplet as a whole remains a quantum object. Amusingly enough, it is this limit where one makes contact with the purely classical Saffman-Taylor fingering. In the next section, we analyse the corresponding large $N$ limit of the matrix integral.

\section{The semiclassical (large $N$ ) limit}

The large $N$ limit we are interested in is $N \rightarrow 0, \hbar \rightarrow 0$ with $\hbar N$ finite and fixed. The expansion in $N^{-1}$ is then the same as the expansion in $\hbar$.

To elaborate the limit, we represent the energy $\mathcal{E}(5)$ in the form

$$
\mathcal{E}=\iint \rho(z) \log \left|z-z^{\prime}\right| \rho\left(z^{\prime}\right) d^{2} z d^{2} z^{\prime}+\frac{1}{\hbar} \int V(z) \rho(z) d^{2} z
$$


where $\rho(z)=\sum_{i} \delta^{(2)}\left(z-z_{i}\right)$ is the density of eigenvalues. In the limit, one treats $\rho(z)$ as a continuous function normalized as $\int \rho(z) d^{2} z=N$. As $\hbar \rightarrow 0$, both terms in (5) are of order $N^{2}$, and the saddle point method can be applied to perform the integral.

The saddle point condition is $\delta \mathcal{E} / \delta \rho(z)=0$ which yields the integral equation for the mean density:

$$
\hbar \int \frac{\rho\left(z^{\prime}\right) d^{2} z^{\prime}}{z-z^{\prime}}+\partial_{z} V(z)=0
$$

The meaning of this equation is especially clear in the Coulomb gas interpretation. It states that each charge is in the equilibrium. Indeed, consider a charge at the point $z$. The first term in the equation is the Coulomb force caused by other charges in the gas while the second term is the external force. The equation just tells that they compensate each other. Clearly, it makes sense to impose the equilibrium condition only in the domain where the charges are actually present, i.e., in the support of eigenvalues.

So, the equation should be satisfied in a domain $D$ where $\rho \neq 0$. Here we assume that $D$ is a connected domain. For example, in the potential $V=-|z|^{2}$ the eigenvalues uniformly fill the disk of radius $\sqrt{\hbar N}$. Small perturbations of the potential slightly disturb the circular shape.

It appears that in case of normal matrices the above integral equation is much easier to solve than the similar equation for distribution of eigenvalues of hermitian matrices. Indeed, on applying $\partial_{\bar{z}}$ to both sides of eq. (7) we obtain $\rho(z)=-\frac{1}{4 \pi \hbar} \Delta V(z)$ in $D$, and $\rho(z)=0$ in $\mathbf{C} \backslash D$. The domain $D$ itself is determined by the condition

$$
\oint_{\partial D} \frac{\partial_{z^{\prime}} V\left(z^{\prime}\right) d z^{\prime}}{z-z^{\prime}}=0
$$

which can be derived from (7) with the help of the Cauchy integral formula. The condition means, in other words, that $D$ is such that the function $\partial_{z} V$ on its boundary is the boundary value of an analytic function in $\mathbf{C} \backslash D$.

An important particular case is $V$ equal to $-|z|^{2}$ plus a harmonic function which we parametrize by its Taylor coefficients $t_{k}$ at the origin: $V=-|z|^{2}+2 \mathcal{R} e \sum t_{k} z^{k}$. Then the density is constant (equal to $\frac{1}{\pi \hbar}$ ) inside $D$ and zero outside. The area of $D$ is equal to $\hbar N$. The shape of $D$ is determined by the conditions

$$
-\frac{1}{\pi k} \int_{\mathbf{C} \backslash D} z^{-k} d^{2} z=t_{k}
$$

which easily follow from (8), so that $\pi k t_{k}$ are harmonic moments of the domain complementary to $D$. In case of quadratic potential the domain is an ellipse [13].

The integrated version of eq. (7) allows one to find the leading contribution to the free energy, which is given by the value of $\hbar^{2} \mathcal{E}$ at the saddle point. Let us denote the latter by $F_{0}$, then

$$
F_{0}=-\frac{1}{16 \pi^{2}} \int_{D} \int_{D} \Delta V(z) \log \left|\frac{1}{z}-\frac{1}{z^{\prime}}\right| \Delta V\left(z^{\prime}\right) d^{2} z d^{2} z^{\prime}
$$

This is the tau-function of curves introduced in [6]. The leading asymptotics of the partition function as $\hbar \rightarrow 0$ is therefore $Z_{N} \simeq e^{F_{0} / \hbar^{2}}$. 


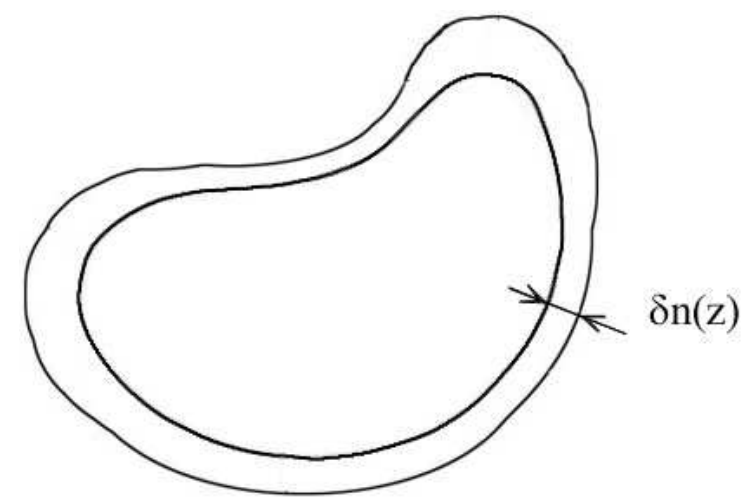

Figure 2: Normal displaycement of the contour.

Small variations of the potential and the growth law. If one varies the potential, $V \rightarrow V+\delta V$, and size of the matrix, $N \rightarrow N+\delta N$, the support of eigenvalues slightly changes its shape and area. Let us examine how it goes. It is natural to characterize an infinitesimal change of the boundary by its normal displaycement $\delta n(z)$ at each point $z$, so that $\delta n(z)$ is a continuous function on the boundary curve (see Fig. 2).

First we vary the potential at constant $N$. The shape of the support of eigenvalues is determined by eq. (8). Its variation can be written as

$$
\oint \frac{\partial_{z^{\prime}} \delta V\left(z^{\prime}\right) d z^{\prime}}{z-z^{\prime}}+\frac{i}{2} \oint \frac{\Delta V\left(z^{\prime}\right) \delta n\left(z^{\prime}\right)}{z-z^{\prime}}\left|d z^{\prime}\right|=0
$$

It is natural to employ the ansatz $\Delta V(z) \delta n(z)=\partial_{n} h(z)$ where $h$ is yet unknown function in the exterior of $D$ such that $h=0$ on the boundary, and $\partial_{n}$ means its normal derivative, with the normal vector pointing outward. This ansatz is suggested by an easy transform of the second integral into a Cauchy integral. Combining the two terms, we get $\oint \frac{\partial_{z^{\prime}}\left(\delta V\left(z^{\prime}\right)+h\left(z^{\prime}\right)\right)}{z-z^{\prime}} d z^{\prime}=0$ for $z \in D$. This implies that the function $\partial_{z}(\delta V+h)$ is analytic in the exterior of $D$, i.e., that the function $\delta V+h$ is harmonic in there. Hence $h(z)=\delta V^{H}(z)-\delta V$ where, given a function $f$, we use the notation $f^{H}$ for the harmonic continuation of this function from the boundary to the exterior of $D$. Therefore,

$$
\delta n(z)=\frac{\partial_{n}\left(\delta V^{H}(z)-\delta V(z)\right)}{\Delta V(z)}
$$

Similarly, to find how the domain $D$ grows at constant $V$ as $N$ increases, we use eq. (8) again. This time the first term in (10) is zero. Noting that

$$
\delta N=-\frac{1}{4 \pi \hbar} \oint \delta n(z) \Delta V(z)|d z|
$$

it is easy to see that the solution is

$$
\delta n(z)=-\frac{2 \hbar \delta N}{\Delta V(z)} \partial_{n} \log |w(z)|
$$

where $w(z)$ is the conformal map from $\mathbf{C} \backslash D$ onto the exterior of the unit circle such that $\infty$ is sent to $\infty$. Since $\log |w|=0$ on the boundary, the normal derivative can be substituted by gradient. Therefore, at $\Delta V=$ const we get the Darcy law (11) (cf. [6]). 


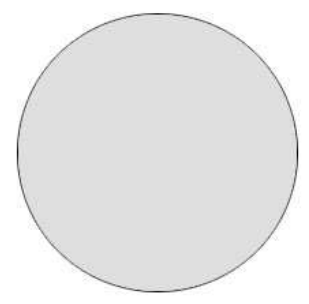

a)

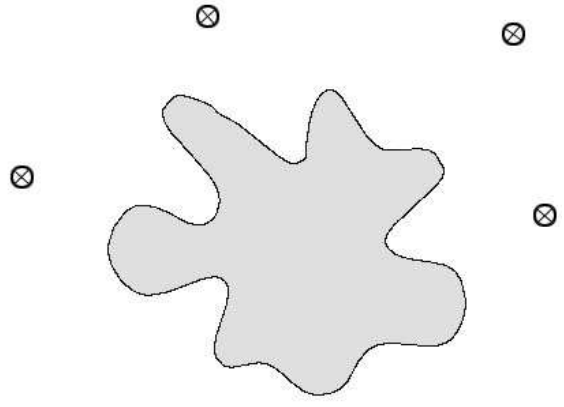

b)

Figure 3: a) The electronic droplet in the uniform magnetic field; b) The electronic droplet in the uniform magnetic field in the peresence of point-like fluxes at the points marked by $\otimes$.

Semiclassical electronic droplet in the presence of magnetic impurities. Let us apply the above results to the semiclassical behaviour of an electronic droplet in a strong magnetic field. The notion of the Quantum Hall droplet [14] implies that the electronic liquid is incompressible, i.e., all states at the lowest energy level are occupied. Therefore, we want the degeneracy of the level to be equal to $N$. This can be achieved in different ways. One of them is to assume the following arrangement. Let a strong uniform magnetic field $B_{0}>0$ be applied in a large disk of radius $R_{0}$. The disk is surrounded by a large annulus $R_{0}<|z|<R_{1}$ with a magnetic field $B_{1}<0$ such that the total magnetic flux through the system is $N \Phi_{0}$. The magnetic field outside the largest disk $|z|<R_{1}$ vanishes. The disk is connected through a tunnel barier to a large capacitor that maintains a small positive chemical potential slightly above the zero energy. If $B_{0}$ is strong enough, the gap is large, and the higher levels can be neglected. In this arrangement, the circular droplet of $N$ electrons is trapped at the center. Its radius is much less than $R_{0}$. The function $V(z)$ for $|z|<R_{0}$ is $V(z)=-\frac{1}{2} B_{0}|z|^{2}$.

Now let us apply a non-uniform magnetic field $\delta B$ somewhere inside the disk $|z|<R_{0}$ but well away from the droplet. Suppose that the nonuniform magnetic field does not change the total flux: $\int \delta B d^{2} z=0$. The potential $V(z)$ inside and around the droplet is modified as

$$
V(z)=-\frac{B_{0}}{2}|z|^{2}-\frac{1}{\pi} \int \log \left|z-z^{\prime}\right| \delta B\left(z^{\prime}\right) d^{2} z^{\prime}
$$

The second term is harmonic inside and around the droplet. One may have in mind thin solenoids carrying magnetic flux ("magnetic impurities"). In the case of point-like magnetic fluxes $q_{i}$ at points $a_{i}$, we have $V(z)=-\frac{1}{2} B_{0}|z|^{2}+\sum_{i} q_{i} \log \left|z-a_{i}\right|$.

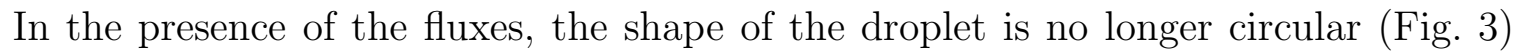
although the magnetic field inside the droplet and not far from it remains uniform and is not changed at all. In this respect this phenomenon is similar to the Aharonov-Bohm effect. The responce of the droplet to an infinitesimal change of the magnetic field $\delta B$ is described by eq. (11) in which

$$
\delta V^{H}(z)-\delta V(z)=\frac{1}{\pi} \int_{\mathbf{C} \backslash D} G\left(z, z^{\prime}\right) \delta B\left(z^{\prime}\right) d^{2} z^{\prime}
$$

Here $G\left(z, z^{\prime}\right)$ is the Green function of the Dirichlet boundary problem in $\mathbf{C} \backslash D$ normalized 
in such a way that $G\left(z, z^{\prime}\right) \rightarrow \log \left|z-z^{\prime}\right|$ as $z \rightarrow z^{\prime}$. In fact this formula holds for arbitrary $\delta B$, not necessarily vanishing inside the droplet. In particular, for small point-like fluxes $\delta q_{i}$ at some points $a_{i}$ we have $\delta V=\sum_{i} \delta q_{i} \log \left|z-a_{i}\right|, \delta B=-\pi \sum_{i} \delta q_{i} \delta^{(2)}\left(z-a_{i}\right)$, and

$$
\delta V^{H}(z)-\delta V(z)=-\sum_{i} G\left(z, a_{i}\right) \delta q_{i}
$$

If $a_{i}$ is inside, $G\left(z, a_{i}\right)$ is set to be zero. The sum, therefore, goes over outside fluxes only. The fluxes inside the droplet, if any, appear to be completely screened and do not have any influence on its shape.

When $B_{1}$ adiabatically increases, with $B_{0}$ and $\delta B$ fixed, the droplet grows because the degeneracy of the lowest level is enlarged and new electrons enter the system. The growth is described by eq. (12) with $\Delta V(z)=-2 B_{0}$ which is equivalent to the Darcy law.

\section{Conclusion}

We have analysed the large $N$ limit of the model of normal random matrices. It has been argued that as $N$ increases, the growth of the support of complex eigenvalues simulates important physical phenomena:

- Interface dynamics in viscous flows (the Saffman-Taylor fingering) in the zero surface tension limit

- Semiclassical behaviour of 2D electronic droplets in the Quantum Hall regime

The former is purely classical while the latter is purely quantum.

The relation to the matrix model may help to suggest a way to regularize singularities which usually occur in the zero surface tension limit and to obtain an analytically tractable formulation of the Saffman-Taylor problem with surface tension.

\section{Acknowledgments}

I am grateful to O.Agam, E.Bettelheim, I.Kostov, I.Krichever, A.Marshakov, M.MineevWeinstein and P.Wiegmann for collaboration. This work was supported in part by the LDRD project 20020006ER "Unstable Fluid/Fluid Interfaces" at Los Alamos National Laboratory during the author's visit at LANL in January-February 2002, by RFBR grant 00-02-16477, and by grant INTAS-99-0590.

\section{References}

[1] M.L.Mehta, Random matrices, Academic Press, NY, 1967

[2] T.Guhr, A.Müller-Groeling and H.Weidenmüller, Phys. Rep. 299 (1998) 189-428, e-print archive: cond-mat/9707301 
[3] P.Di Francesco, P.Ginsparg and J.Zinn-Justin, Phys. Rep. 254 (1995) 1-133

[4] Y.Fyodorov, B.Khoruzhenko and H.-J.Sommers, Phys. Rev. Lett. 79 (1997) 557, e-print archive: cond-mat/9703152; J.Feinberg and A.Zee, Nucl. Phys. B504 (1997) 579-608, e-print archive: cond-mat/9703087; G.Akemann, e-print archive: hepth/0204246

[5] D.Bensimon, L.P.Kadanoff, S.Liang, B.I.Shraiman, and C.Tang, Rev. Mod. Phys. 58 (1986) 977

[6] M.Mineev-Weinstein, P.B.Wiegmann and A.Zabrodin, Phys. Rev. Lett. 84 (2000) 5106-5109, e-print archive: nlin.SI/0001007; I.Kostov, I.Krichever, M.MineevWeinstein, P.Wiegmann and A.Zabrodin, $\tau$-function for analytic curves, Random matrices and their applications, MSRI publications, eds. P.Bleher and A.Its, vol.40, p. 285-299, Cambridge Academic Press, 2001, e-print archive: hep-th/0005259

[7] O.Agam, E.Bettelheim, P.Wiegmann and A.Zabrodin, Phys. Rev. Lett. 88 (2002), 236801, e-print archive: cond-mat/0111333; P.Wiegmann, in: Statistical Field Theories, pp. 337-349, A. Cappelli and G. Mussardo (eds.), Kluwer Academic Publishers, 2002, e-print archive: cond-mat/0204254

[8] P.B.Wiegmann and A.Zabrodin, Commun. Math. Phys. 213 (2000) 523-538, eprint archive: hep-th/9909147; A.Zabrodin, Teor. Mat. Fiz. 129 (2001) 239-257 (in Russian, English translation: Theor. Math. Phys. 129 (2001) 1511-1525), eprint archive: math.CV/0104169; A.Gorsky, Phys. Lett. B498 (2001) 211, e-print archive: hep-th/0010068; A.Marshakov, P.Wiegmann and A.Zabrodin, Commun. Math. Phys. 227 (2002) 131-153, e-print archive: hep-th/0109048

[9] L.-L.Chau and Y.Yu, Phys. Lett. 167A (1992) 452; L.-L.Chau and O.Zaboronsky, Commun. Math. Phys. 196 (1998) 203-247, e-print archive: hep-th/9711091

[10] J.-M.Daul, V.Kazakov and I.Kostov, Nucl. Phys. B409 (1993) 311-338, e-print archive: hep-th/9303093; M.Adler and P. van Moerbeke, Ann. of Math. (2) 149 (1999), no. 3, 921-976, e-print archive: hep-th/9907213

[11] F.J.Dyson, J. Math. Phys. 3 (1962) 140

[12] Y.Aharonov and A.Casher, Phys. Rev. A 19 (1979) 2461; J.E.Avron and R.Seiler, Phys. Rev. Lett. 42 (1979) 931

[13] P.Di Francesco, M.Gaudin, C.Itzykson and F.Lesage, Int. J. Mod. Phys. A9 (1994) 4257-4351

[14] R.B.Laughlin, in: The Quantum Hall Effect, R.E.Prange and S.M.Girvin (eds.), Springer, New York, 1987; S.Iso, D.Carabali and B.Sakita, Phys. Lett. B296 (1992) 143; A.Cappelli, C.Trugenberger and G.Zemba, Nucl. Phys. B396 (1993) 465 